\title{
Infarto do miocárdio e acidente vascular cerebral associados à alta temperatura e monóxido de carbono em área metropolitana do sudeste do Brasil
}

\author{
Miocardic infarcts and cerebral-vascular accidents associated with high \\ temperature and carbon monoxide in an metropolitan area of Southeastern Brazil
}

\author{
Davi Rumel*, Lucia Ferreira Riedel*, Maria do Rosario D. O. Latorre*, Bruce Bartholow Duncan**
}

\begin{abstract}
RUMEL, D. et al. Infarto do miocárdio e acidente vascular cerebral associados à alta temperatura e monóxido de carbono em área metropolitana do sudeste do Brasil. Rev. Saúde Pública, 27: 15-22, 1993. Muito se tem publicado e discutido acerca de fatores de risco ligados a estilo de vida e a fatores hereditários em Acidente Vascular Cerebral (AVC) e Infarto do Miocárdio (IM). Porém o estudo da influência de fatores ambientais como poluição por monóxido de carbono e temperatura na determinação nas ocorrências dessas patologias ainda é pouco discutido em nosso meio. Visando preencher esta lacuna, foi verificada a associação existente entre os valores de temperaturas máximas diárias e valores médio e máximo diários de monóxido de carbono e AVC e IM no Município de São Paulo, SP (Brasil). Foi coletada uma série histórica do número de casos novos de AVC e IM atendidos em Pronto Socorro de um hospital de clínicas e valores de temperatura e monóxido de carbono. Conclui-se que das internaçőes anuais por $\mathrm{IM}, 2,1 \%$ são devidos à poluição atmosférica e $4,9 \%$ a altas temperaturas. Das internações anuais por AVC, $2,8 \%$ são devidas a altas temperaturas. Não foi identificado associação entre monóxido de carbono e AVC.
\end{abstract}

Descritores: Infarto do miocárdio, epidemiologia. Distúrbios cerebrovasculares, epidemiologia. Calor, efeitos adversos. Monóxido de carbono, efeitos adversos.

\section{Introduçăo}

Com a transição epidemiológica ocorrida neste século, através do controle das doenças transmissíveis, observou-se aumento da esperança de vida em todos os países. Este fato faz com que as doenças não-transmissíveis passem a ter importância maior quando se pensa em morbidade e mortalidade.

Dentre as doenças não-transmissíveis interessam, particularmente, os acidentes cardiovasculares cerebrais (AVC) e o infarto do miocárdio (IM) devido à sua importância no obituário geral, tanto em homens quanto nas mulheres, e pelos altos custos requeridos no seu tratamento e na reabilitação dos pacientes acometidos por esse mal.

\footnotetext{
* Departamento de Epidemiologia da Faculdade de Saúde Pública da Universidade de São Paulo - São Paulo, SP Brasil.

** Faculdade de Medicina da Universidade Federal do Rio Grande do Sul - Porto Alegre, RS - Brasil.
}

Separatas/Reprints: 208-3846 Carrigan Court - Bumaby B. C. V3N 4H9 - Canada.

Edição subvencionada pela FAPESP. Processo Medicina 93/ 0208-5.
Neste sentido é importante que se estude quais seriam os seus fatores de risco para que se possa planejar, adequadamente, medidas de prevenção e controle deste grupo de doenças.

Muito se tem publicado e discutido acerca dos fatores de risco ligados a estilo de vida e a fatores hereditários, porém o estudo da influência dos fatores ambientais como poluição e temperatura na ocorrência tanto do AVC quanto do IM é assunto pouco discutido no Brasil.

O poluente mais relacionado com AVC e IM é o monóxido de carbono ( $\mathrm{CO}$ ), cujas fontes principais são a fumaça de cigarro e a de veículos a motor.

Quando se pensa em poluição do ar não se pode esquecer de estudar, conjuntamente, os efeitos da temperatura. Este controle é fundamental, pois a queda de temperatura pode ser acompanhada do fenômeno de inversão térmica, que provoca aumento da concentração de poluentes no ar.

Em 1912 Haldane observou que uma mistura de gás de 245 moléculas de oxigênio e 12 de monóxido de carbono dão igual proporção de oxihemoglobina e carboxihemoglobina. O equilíbrio desta saturação é extremamente rápida. Quando a tensåo de carboxihemoglobina ultrapassa $40 \%$ pode haver um colapso circulatorio (Goldsmith \& Aronow', 1975). 
Entre os órgãos do corpo, o músculo cardíaco é o que mais extrai oxigênio. Em pessoas com processo arteriosclerótico, esta necessidade, aliada a altas concentrações de carboxihemoglobina, faz o coração ser muito vulnerável à isquemia (Goldsmith \& Aronow $\left.{ }^{9}, 1975\right)$. Mesmo pequenas elevações de $C O$ sanguínea são suficientes para produzir adaptações fisiológicas no sistema circulatório que levam a um aumento do trabalho cardíaco entre outras alterações (Ayres e col. ${ }^{4}$, 1969). Wanstrup e col. ${ }^{17}$, 1969), realizaram estudos em coelhos e concluíram que há uma elevação na produção de ateromas naqueles expostos a altas taxas de $\mathrm{CO}$ atmosférico.

Hexter \& Goldsmith ${ }^{10}, 1971$, encontraram uma correlação significante $(p<0,002)$ entre concentração de $\mathrm{CO}$ e a mortalidade geral, ajustada pela temperatura, na cidade de Los Angeles.

Aronow e col.2, 1972, em estudo realizado com 10 pacientes com angina, observaram em todos uma diminuição da tolerância ao exercício físico após dirigirem em ruas de áreas poluídas. $O$ mesmo não ocorria quando dirigiam em áreas nãopoluidas.

Existe equilíbrio homeostático de manter a temperatura do corpo constante, mesmo que varie a temperatura externa. Porém, este mecanismo torna-se menos eficiente com 0 avançar da idade e são os idosos as principais vítimas dos extremos de temperatura, pois a taxa metabólica (que produz calor) neles é baixa. O sistema sensorial diminui, a resposta vasomotora é retardada e o mecanismo de sudorese não funciona adequadamente (Collins \& Exton-Smith ${ }^{6}, 1983$ ).

O aumento brusco de temperatura provoca um suor severo que leva a uma redução do volume plasmático e queda da pressão arterial. Além disso há aumento na viscosidade do sangue, da concentração de colesterol e do número de hemácias e de plaquetas. A queda da pressão reduz o fluxo nas arteríolas e as plaquetas, em número elevado na presença de colesterol, facilitam o aparecimento de trombos. Estes envolvem-se em ateromas e interrompe o fluxo sangüíneo para os tecidos, causando, clinicamente, IM ou AVC, conforme 0 órgão afetado (Keatinge e col. ${ }^{11}, 1986$ ).

Rogot \& Radgett ${ }^{13}, 1976$, foram os primeiros a definir uma faixa de temperatura ideal para doenças do aparelho cardio-circulatório. A partir das informaçōes de mortalidade, padronizadas por idade, de 32 áreas metropolitanas dos Estados Unidos, concluíram que em regioes frias a faixa de temperatura ideal é entre $15,6^{\circ} \mathrm{C}$ e $26,6^{\circ} \mathrm{C}$. Abaixo ou acima dessas temperaturas tanto a mortalidade por AVC quanto por IM tendem a aumentar exponencialmente. Quando ocorrem ondas de calor em regiōes frias, há aumento na mortalidade por doenças cardiovasculares, principalmente em idosos. Em regióes quentes, a faixa de temperatura ideal é entre $26,7^{\circ} \mathrm{C}$ e $32^{\circ} \mathrm{C}$.

Há outras contribuições relevantes nessa área. Ohno $^{12}$, 1969, em estudo de 1.642 óbitos por AVC, em Nagoya, Japão, encontrou um significativo excesso de morte em expostos aos seguintes fatores atmosféricos: baixa velocidade do ar, baixos e altos índices de temperatura-umidade, alta umidade do ar e baixa pressão atmosférica.

Ellis 7 , 1972, mostra que a mortalidade, por ocasião das ondas de calor, começou a diminuir após a segunda guerra mundial, possivelmente devido à expansão do uso de ar condicionado. Além da associação com os idosos, já relatada por outros autores, identificou que os negros têm duas vezes maior risco de morte do que os brancos, por ocasião das ondas de calor.

Em Londres, onde "smog", inversão térmica e poluição ambiental são palavras associadas às primeiras epidemias agudas de doenças nãotransmissíveis, ainda na década de 50, Tout ${ }^{16}$, 1980, mostrou que o excesso de mortalidade em dias sem "smog", sem inversão térmica e sem poluição, mas em um período de onda de calor, era devido ao IM, AVC e às doenças respiratórias.

$O$ papel de ventos quentes e secos no aumento da incidência de AVC, na província de Brescia (Itália) e no aumento de IM e AVC, no Kwait, foram relatados, respectivamente, por Saviola e col.15, 1986 e Al-Yusuf e col. ${ }^{1}, 1986$.

A faixa de temperatura ideal também foi objeto de estudo mais recente, quando Auciliems \& Skinner ${ }^{3}, 1989$, analisaram a mortalidade por doença cardiovascular em Brisbane (Austrália), cidade esta sem grandes extremos de temperatura, e concluíram que a faixa de temperatura ideal ficava entre $18,0^{\circ} \mathrm{C}$ e $24,0^{\circ} \mathrm{C}$.

Berginer e col. ${ }^{5}, 1989$, estudaram a associação entre temperatura e admissão hospitalar por AVC, em um hospital em Israel, constatando um risco relativo de 2,76 entre dias quentes e dias frios agregados.

No Brasil, há indícios de associação entre calor e mortalidade por doença cardiovascular. A região do noroestę paulista é a região mais quente do Estado e é onde se localizam as cidades de Ribeirão Preto e Bauru que apresentam as maiores mortalidades por AVC para o Estado8. Porém, o mesmo não se verifica com o IM. Rumel ${ }^{14}$, identificou associação entre a mortalidade por AVC e trabalhadores masculinos da agricultura, que é a base principal da economia do noroeste paulista.

Faltam estudos específicos para averiguaar se as associações já identificadas em outros países também ocorrem entre nós. No presente trabalho objetiva-se verificar a associação existente entre fa- 
tores ambientais e a incidência de AVC e IM no Município de São Paulo.

\section{Materlal e Método}

\section{Material de Estudo}

Foi coletada série histórica com aferição retrospectiva do número de casos novos de AVC e de IM atendidos diariamente nos Pronto-Socorros do Instituto Central e Instituto do Coração do Hospital das Clínicas da Faculdade de Medicina da Universidade de São Paulo (USP). Estes dados foram obtidos a partir do banco de dados de morbidade hospitalar do Centro de Informações de Saúde da Secretaria de Estado da Saúde.

Não foi considerada a origem do paciente, pois trata-se de casos agudos e a exposição no dia pode atingir também aqueles que não moram no $\mathrm{Mu}$ nicípio de São Paulo, mas que, por algum motivo, nele se encontravam.

Foram incluídas todas as pessoas com diagnóstico confirmado, pois o monóxido de carbono e a temperatura máxima desencadeiam uma série de ajustes compensatórios no sistema vascular, independente do sexo e idade.

Foi coletada na CETESB (Companhia de Tecnologia e Saneamento Ambiental do Estado de São Paulo), série histórica com aferição retrospectiva dos valores de temperatura e monóxido de carbono.

Os dados de temperatura têm como local de amostragem a estação "Centro" e os de $\mathrm{CO}$ as estaçōes "Cerqueira César" e "Praça do Correio", no centro de São Paulo.

Para os dados de $\mathrm{CO}$ foram utilizadas duas estaçōes devido a ausência de coleta desses dados em diferentes dias. Quando se coletaram dados nas duas estações utilizou-se média aritmética dos mesmos.

\section{Delineamento}

Trata-se de delineamento ecológico, que se caracteriza pelo fato da unidade de estudo ser uma medida de tempo ou localização geográfica em vez da unidade de estudo-pessoa, como é o usual nos demais delineamentos epidemiológicos. No caso, a unidade de estudo é o dia.

Como não se trata de estudo que visa a identificar a influência dos fatores ambientais no processo arterioesclerótico e, sim, a influência desses fatores na determinação do momento da agudização deste processo, variáveis como sexo, idade, hipertensão arterial e hipercolesterolenemia não foram consideradas como variáveis de confusão. Uma possível variável desta categoria, neste momento da história natural dessas doenças, seria a intoxicação por monóxido de carbono devido ao hábito de fumar. Porém, como não se acredita existir associação entre o hábito de fumar e a concentração de monóxido de carbono, a ausência dessa informação não prejudicou a análise dos resultados. Optou-se por não analisar outros fenômenos meteorológicos, pois quando aparecem na literatura, estão associados a alteraçōes de temperatura e concentraçōes de monóxido de carbono.

\section{Período de Estudo}

Optou-se por um período de dois anos para se avaliar a possivel sazonalidade dos aspectos estudados. Foram coletadas informações registradas entre 1 de abril de 1989 a 31 de março de 1991, últimos dois anos disponíveis nas fontes consultadas.

\section{Variáveis de Estudo}

As variáveis estudadas foram os casos novos de AVC e de IM, como dependentes; e temperatura máxima diária (em graus Celsius); média diária de CO (em partes por milhão), valor médio dos oito maiores valores em 24 horas; concentração máxima diária de uma hora de $\mathrm{CO}$ (em partes por milhão), como independentes.

\section{Análise}

Foi feita a distribuição temporal dos casos de IM e AVC em dois anos consecutivos, seguida da análise de regressão para cada um dos fatores ambientais em estudo e o número de casos, através de regressão múltipla, verificando as possíveis interaçōes e confundimentos. Para os fatores ambientais que se mostraram significantes nas análises anteriores, foi feita uma transformação nas mesmas originando variáveis "dummy".

O nivel de significância foi de alfa igual a 5\%.

$O$ "software" utilizado nas análises dos dados foi o Epi Info (versão 5.0).

\section{Resultados}

Nos dois anos de estudo foram identificados 7.798 casos de IM e 4.654 casos de AVC, que não apresentaram sazonalidades como pode ser observado pelos gráficos das Figuras 1 a 4 .

\section{Associação com Infarto do Miocárdio:}

Ao se realizar a análise da regressão linear simples de casos de IM e a temperatura máxima ob- 


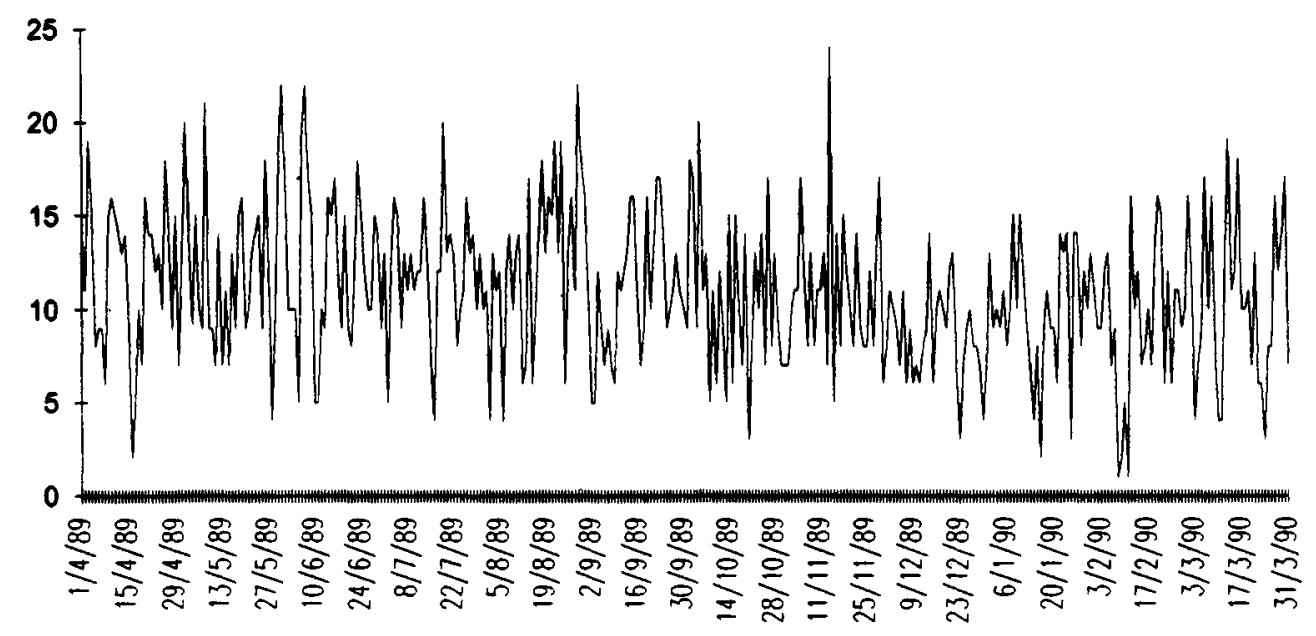

Flgura 1. Casos de internação por infarto do miocárdio no Hospital daś Clínicas da FMUSP entre 1-4-89 a 31-3-90.

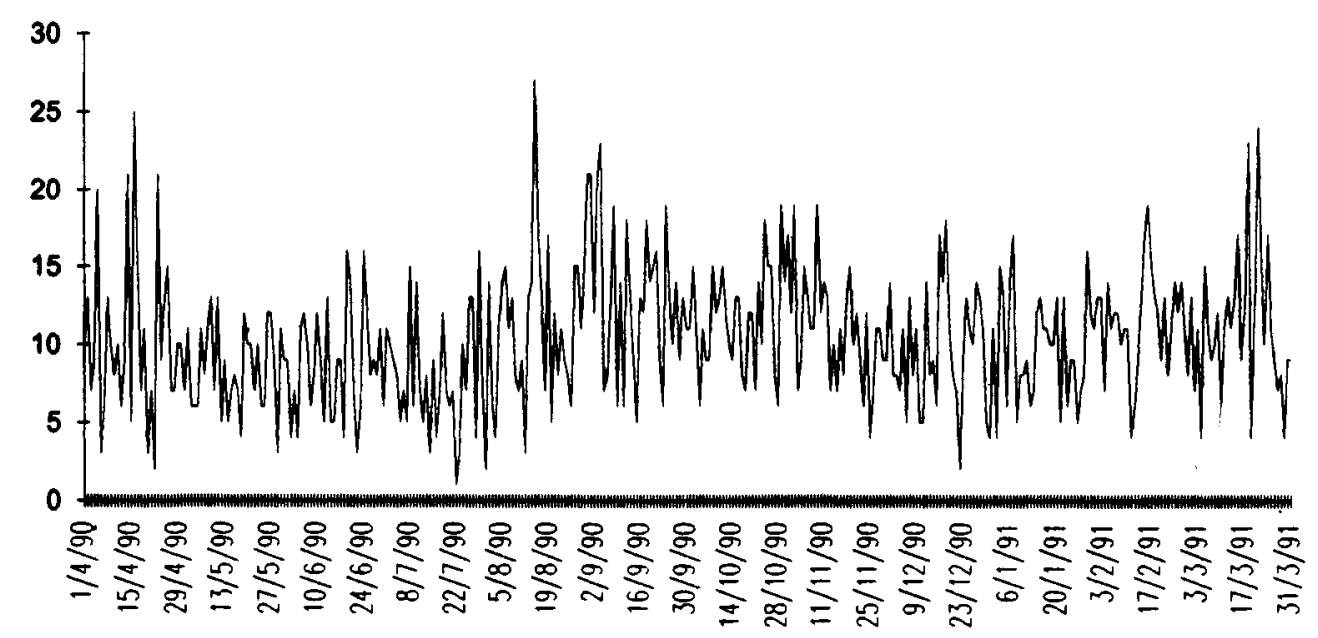

Flgura 2. Casos de internação por infarto do miocárdio no Hospital das Clínicas da FMUSP entre 1-4-89 a 31-3-90.

teve-se um coeficiente de correlação igual a 0,12 , com um intervalo de confiança $(95 \%)$ igual a $(0,05 ; 0,20)$. Apesar do pouco poder explicativo do modelo $\left(r^{2}=0,02\right)$, verifica-se que o coeficiente de correlação é significante, o que mostra serem as duas variáveis correlacionadas.

Ao acrescentar-se no modelo linear o termo quadrático (temperatura máxima) ${ }^{2}$ obteve-se que esta parcela não era estatisticamente significante ao nível de alfa $=5 \%$, o que demonstra ser 0 modelo linear o mais adequado para se verificar o relacionamento entre número de casos de IM (y) e temperatura máxima (X). O modelo obtido foi:

$y=8,06+0,10 x$
A variável temperatura máxima foi categorizada em 5 extratos com a criação de 4 variáveis "dummy", a saber:

Temperatura máxima

$$
\begin{array}{llll}
X_{1} & X_{2} & X_{3} & X_{4}
\end{array}
$$

$\begin{array}{rllll}<=20^{\circ} \mathrm{C} & 0 & 0 & 0 & 0 \\ 21-24^{\circ} \mathrm{C} & 1 & 0 & 0 & 0 \\ 25-26^{\circ} \mathrm{C} & 0 & 1 & 0 & 0 \\ 27-29^{\circ} \mathrm{C} & 0 & 0 & 1 & 0 \\ >=30^{\circ} \mathrm{C} & 0 & 0 & 0 & 1\end{array}$

Obtendo o seguinte modelo estimado:

$$
y=9,53+1,26 x_{1}+0,70 x_{2}+1,80 x_{3}+1,45 x_{4}
$$




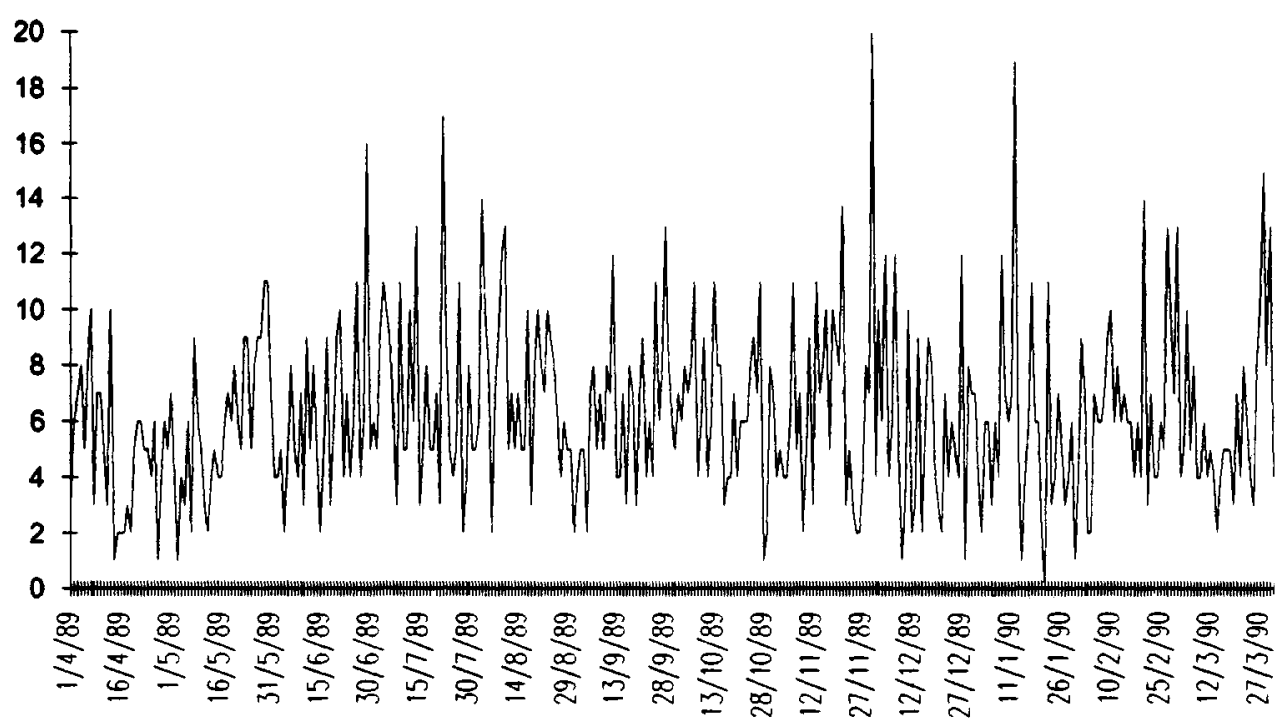

Figura 3. Casos de internação por acidente vascular cerebral no hospital das Clínicas da FMUSP entre 1-489 a $31-3-90$.

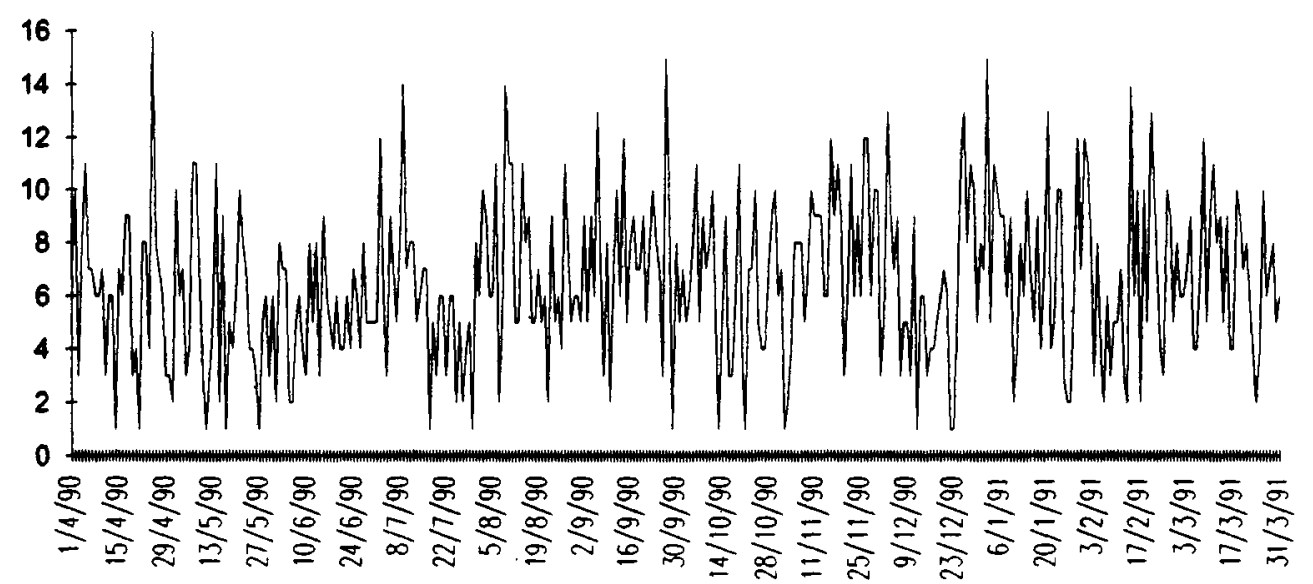

Figura 4. Casos de internação por acidente vascular cerebral no Hospital das Clínicas da FMUSP entre 1-490 a 31-3-91.

sendo que somente $X_{3}$ foi não significante $(p>0,05)$.

Optou-se por novo modelo com apenas três extratos originando apenas duas variáveis "dummy":

Temperatura máxima

$$
\mathrm{X}_{1} \quad \mathrm{X}_{\mathbf{2}}
$$

\begin{tabular}{|c|c|}
\hline $\begin{array}{r}<=20^{\circ} \mathrm{C} \\
21-26^{\circ} \mathrm{C} \\
>=27^{\circ} \mathrm{C}\end{array}$ & $\begin{array}{l}0 \\
1 \\
0\end{array}$ \\
\hline
\end{tabular}

Obteve-se, então, o scguinte modelo final:
$Y=9,53+1,06 X_{1}+1,61 X_{2}$, com todos os betas significantes.

Mesmo procedimento foi adotado em relação à variável independente concentração máxima de monóxido de carbono em 1 hora (CO-1h).

A análise de regressão para o modelo linear de casos de IM (Y) e CO-1h (Z) obteve um coeficiente de correlação igual a 0,14 com um intervalo de confiança $(95 \%)$ igual a $(0,07 ; 0,21)$. Apesar do pouco poder explicativo do modelo $\left(\mathrm{r}^{2}=0,02\right)$, a mesma situação encontrada em relação à temperatura máxima aqui se verifica: o coeficiente de cor- 
relação é diferente de zero o que indica que as duas variáveis são correlacionadas. Ao se acrescentar 0 termo quadrático não houve melhora na precisão do modelo, optando-se, então, por manter apenas o termo da equação linear. O modelo obtido foi:

$\mathrm{Y}=9,13+0,13 \mathrm{Z}$

Tendo em vista que CO-1h (Z) poderia ser uma possível variável de confundimento de temperatura (X), estimou-se um modelo com as duas variáveis com o seguinte resultado:

$Y=7,57+0,08 X+0,08 Z$

Observa-se que os valores dos coeficientes angulares tanto de $\mathrm{X}$ quanto de $\mathrm{Z}$ diminuíram, mas não o suficiente para eliminar-se alguma destas variáveis do modelo. Isso mostra que há um confundimento entre as mesmas.

A seguir inclui-se no modelo o termo de interação (XZ), mas não foi significante.

A variável $\mathrm{CO}-1 \mathrm{~h}$ foi categorizada em 5 extratos, criando-se 4 variáveis "dummy", demostradas a seguir:

$\begin{array}{cllll}\text { CO-1h (ppm) } & \mathrm{Z}_{1} & \mathrm{Z}_{2} & \mathrm{Z}_{3} & \mathrm{Z}_{4} \\ <=8,00 & 0 & 0 & 0 & 0 \\ 8,05-10,00 & 1 & 0 & 0 & 0 \\ 10,05-12,00 & 0 & 1 & 0 & 0 \\ 12,05-16,00 & 0 & 0 & 1 & 0 \\ >=16,05 & 0 & 0 & 0 & 1\end{array}$

O modelo obtido foi:

$$
Y=10,31+0,01 Z_{1}-0,41 Z_{2}+0,63 Z_{3}+1,53 Z_{4}
$$

Os únicos betas significamente diferentes de zero foram os correspondentes a $Z_{3}$ e a $Z_{4}$.

Optou-se por uma nova extratificação com a criação de duas variáveis "dummy":

$\begin{array}{cll}\text { CO-1h (ppm) } & Z_{1} & Z_{2} \\ <=12,00 & 0 & 0 \\ 12,05-16,00 & 1 & 0 \\ >=16,05 & 0 & 1\end{array}$

Obteve-se o seguinte modelo final com todos os betas significantes:

$$
Y=10,19+0,76 Z_{1}+1,66 Z_{2}
$$

A seguir verificou-se o confundimento entre as variáveis $Z_{1}$ e $Z_{2}$ referentes ao modelo final de CO-1h e $X_{1}$ e $X_{2}$ ao modelo final de temperatura máxima. $O$ modelo resultante foi:

$$
Y=9,41+0,83 X_{1}+1,28 X_{2}+0,57 Z_{1}+1,38 Z_{2}
$$

Neste último modelo apenas os betas referentes a $X_{2}$ e $Z_{2}$ foram significantes $(p<0,05)$.

Em relação à última variável independente, média de monóxido de carbono em 8 horas - CO-8h, foi feita análise semelhante a das outras variáveis anteriores.

Foi verificada a possível correlação entre $\mathrm{CO}$ $8 \mathrm{~h}(\mathrm{~W})$ e casos de IM (Y). Obteve-se um coeficiente de correlação igual a 0,11 com um intervalo de confiança igual a $(0,04 ; 0,19)$. Apesar do baixo poder explicativo $\left(r^{2}=0,01\right)$, a correlação é significantemente diferente de 0 . Com a inclusão do termo quadrático $\left(\mathrm{W}^{2}\right)$ no modelo de regressão linear simples, este termo não apresentou significância estatística, sendo o modelo obtido, o seguinte:

$\mathrm{Y}=9,22+0,16 \mathrm{~W}$

A análise do confundimento entre $\mathrm{CO}-8 \mathrm{~h}$ e temperatura máxima apresentou 0 seguinte resultado:

$$
\mathrm{Y}=7,51+0,09 \mathrm{X}+0,10 \mathrm{~W}
$$

$O$ beta referente ao $\mathrm{CO}-8 \mathrm{~h}$ foi não-significante. Portanto, não foi considerado $\mathrm{CO}-8 \mathrm{~h}$ no modelo final.

\section{Associaçāo com Acidente Vascular Cerebral}

Ao se realizar a análise da regressão linear simples de casos de AVC e a temperatura máxima, obteve-se um coeficiente de correlação igual a $0,08(0,00 ; 0,16)$. Apesar do pouco poder explicativo do modelo $\left(\mathrm{r}^{2}=0,01\right)$ e do intervalo de confiança incluir o valor zero, optou-se por manter esta variável. Ao se acrescentar o termo quadrático não houve melhora na precisão do modelo, decidindose, então, por manter a equação linear. $O$ modelo obtido foi:

$$
Y=5,22+0,05 X
$$

A variável temperatura máxima foi categorizada em 5 extratos, criando-se 4 variáveis "dummy", em procedimento análogo à análise com IM. O modelo estimado foi o seguinte:

$$
Y=5,97+0,28 X_{1}+0,26 X_{2}+0,87 X_{3}+0,62 X_{4}
$$

$O$ único beta significantemente diferente de zero foi o correspondente a $\mathrm{X}_{3}$. Optou-se por uma nova extratificação com a criação de duas variáveis "dummy": 
Temperatura máxima

$\begin{array}{rll}<=20^{\circ} \mathrm{C} & 0 & 0 \\ 21-26^{\circ} \mathrm{C} & 1 & 0 \\ >=27^{\circ} \mathrm{C} & 0 & 1\end{array}$

Obteve-se o seguinte modelo final com somente $\mathrm{o}$ beta de $\mathrm{X}_{2}$ significante:

$$
Y=5,97+0,28 X_{1}+0,74 X_{2}
$$

A análise de regressão linear simples de casos de AVC e concentração máxima de monóxido de carbono e 1 hora (CO-1h) resultou em um modelo não significante, excluindo-se esta variável de análises subsequientes. $O$ mesmo ocorreu com a análise de casos de AVC com concentração média de monóxido de carbono em 8 horas (CO-8h).

\section{Discussão}

De acordo com o modelo final para IM, $\gamma=$ $9,41+0,83 X_{1}+1,28 X_{2}+0,57 Z_{1}+1,38 Z_{2}$, apenas $X_{2}$ e $Z_{2}$ foram significantes. Por se tratar de variáveis "dummy", as $X_{1}$ e $Z_{1}$ não foram excluídas do modelo final pois, se o fossem, estariam sendo alterados os extratos de referência.

A condição de $\mathrm{X}_{2}=1$, que representa temperaturas máximas maiores que $26^{\circ} \mathrm{C}$, corresponde a $41 \%$ dos dias do ano (150 dias), no período estudado. Nesses dias o número médio de casos de IM, internados no Pronto-Socorro do Hospital das Clínicas, elevou-se de 9,41 para 10,69 (1,28 vezes a mais) considerando-se que o $\mathrm{CO}-1 \mathrm{~h}$ estava abaixo de $16,05 \mathrm{ppm}$. Isso demonstra um aumento de 192 casos por ano $(1,28 \times 150)$ ou $4,9 \%$ devido a temperatura elevada.

O mesmo raciocínio pode ser feito para a condição $Z_{2}=1$ (supondo-se que a temperatura está abaixo de $27^{\circ} \mathrm{C}$ ), que representa $\mathrm{CO}-1 \mathrm{~h}$ acima de $16,00 \mathrm{ppm}$. Em 16\% dos dias do ano (58 dias) esta condição é observada, o que representa aumento médio de 1,38 casos $(10,79)$ no número médio de 9,41 casos de IM por dia. Isso demonstra um aumento de 80 casos por ano $(1,38 \times 58)$ ou $2,1 \%$ devido ao $\mathrm{CO}-1 \mathrm{~h}$ elevado.

De acordo com o modelo final para AVC, $\gamma=$ $5,97+0,28 X_{1}+0,74 X_{2}$, sendo $X_{2}$ significante, tem-se que temperaturas máximas maiores de $26^{\circ} \mathrm{C}$ correspondem a $41 \%$ dos dias do ano (150 dias), no período estudado. Nesses dias o número médio de casos de AVC internados no Pronto-Socorro do Hospital das Clínicas elevouse acima de 5,97 para 6,71. O aumento de 0,74 casos por dia corresponde a 111 casos a mais por ano, ou $2,8 \%$.

\section{Conclusóes}

Se as pessoas que são atendidas nos ProntosSocorros do Hospital das Clínicas da Faculdade de Medicina da USP são uma parcela do conjunto de casos de IM, que ocorrem na cidade de São Paulo, pode-se concluir que $2,1 \%$ das internações anuais por IM são devidas a poluição atmosférica e 4,9\% das internações anuais são devidas a altas temperaturas.

Poluição atmosférica por monóxido de carbono e calor são fatores de risco ao episódio agudo de IM. Sabe-se que o IM incide em população adulta em idade produtiva e que seu tratamento e reabilitação são caros. O custo aumenta se levarmos em consideração os dias afastados do trabalho por esta emergência. Apesar do impacto da redução deste fator de risco ser pequeno, se menosprezados, a tendência será de aumentar sua importância se os niveis de poluição continuarem a crescer.

Para os casos de AVC, se as pessoas que são atendidas no Hospital das Clínicas da Faculdade de Medicina da USP são uma parcela do conjunto dos que ocorrem na cidade de São Paulo, podemos concluir que $2,8 \%$ das internaçōes anuais por AVC são devidas a altas temperaturas.

Podemos recomendar que pacientes com processo arterioesclerótico, em risco de IM ou AVC, principalmente idosos, devem precaver-se de praticar exercícios e se expor ao sol em dias de altas temperaturas e altas concentrações de monóxido de carbono no ar.

\section{Agradecimentos}

À Eliane Benedictis e Samuel Goihman do Centro de Informações de Saúde da Secretaria de Estado da Saúde do Estado de São Paulo, pelo apoio na coleta de dados.

RUMEL, D. et al. [Miocardic infarcts and cerebralvascular accidents associated wild high temperature and carbon monoxide in an metropolitan area of Southeastem Brazil]. Rev. Saúde Pública, 27: 15-22, 1993. Many studies have been published about the relationship between life style and genetic risk factors and stroke and heart attack, but there have been few about the relationship between atmospheric pollution, specifically with carbon monoxide, and temperature and stroke and heart attack. With a view to filling this gap the relationship between values of carbon monoxide concentration in the air, maximum and average by day, and maximum temperature by day and the number of new cases of CVA and IM admitted as emergencies at the biggest hospital complex of S. Paulo city was analysed. If was concluded, by the use of multivariable regression analysis, that 
$2.1 \%$ and $4.9 \%$ of heart attack admissions were due to carbon monoxide air pollution and high temperature, respectively. $2.8 \%$ of stroke admissions were due high temperature. Stroke and carbon monoxide were not associated in this population.

Keywords: Myocardial infarction, epidemiology. Cerebrovascular disorders, epidemiology. Heat, adverse effects. Carbon monoxide, adverse effects.

\section{Referéncias Bıbllográflcas}

1. AL-YUSUF, A. R.; KOLAR, J.; BHATNAGAR, S. K.; HUDAK, A.; SMII, J. Seasonal variation in the incidence of unstable angina and acute myocardial infarction: effect of dry hot climate on the occurrence of complications following acute myocardial infarction. J. Trop. Med. Hyg., 89: 157-61, 1986.

2. ARONOW, W. S.; ROKOW, S. N.; HARRIS, C. N; ISBELL, M. W.; IMPARATO, B. Effect of freeway travel on angina pectoris. Ann. Intern. Med., 77: 669-76, 1972.

3. AULICIEMES, A \& SKINNER, J. L. Cardiovascular deaths and temperature in subtropical Brisbane. Int. J. Biometeorol., 33: 215-21, 1989.

4. AYRES, S. M.; MUELLER, H. S.; GREGORY, J. J.; GLANNELLI JR., S.; PENNY, J. L. Systemic and myocardial hemodynamic responses to relatively small concentrations of carboxyhemoglobin (COHB). Arch. Environ. Health, 18: 699-709, 1969.

5. BERGINER, V. M.; GOLDSMITH, J.; BATZ, U.; VARDI, H.; SHAPIRO, Y. Clustering of strokes in association with meteorologic in the Negev Desert of Israel: 19811983. Stroke, 20: 65-9, 1989.

6. COLIINS, K. J. \& EXTON-SMITH, A. N. Thermal homeostasis in old age. J. Am. Geriat. Soc., 31: 519-24, 1983.

7. ELIIS, F. P. Mortality from heat illness and heataggravated illness in the United States. Environ. Res., 5: $1-58,1972$.
8. FUNDAÇÃO SEADE. Causas de morte e esperança de vida ao nascer no Estado de Sáo Paulo e regiōes, 1975 . 1983. São Paulo, 1990. (Coleção Realidade Paulista).

9. GOLDSMITH, J. R. \& ARONOW, W. S. Carbon monoxide and coronary heart disease: a review. Environ. Res., 10: 236-48, 1975.

10. HEXTER, A. C. \& GOLDSMTTII. J. R. Carbon monoxide: association of community air pollution with mortality. Science, 172: 265-7, 1971.

11. KEATINGE, W. R.; COLESHAW, S. R.; EASTON, J. C.; COTTER, F; MATTOCK, M. B.; CHELLIAH, R. Increased platelet and red cell counts, blood viscosity and plasma cholesterol levels during heat stress and mortality from coronary and cerebral thrombosis. Am. J. Med., 81: 795-800, 1986.

12. OHNO, Y. Biometeorologic studies on cerebrovascular diseases: effects of meteorologic factors on the death from cerebrovascular accident. Jpn. Circ. J., 33: 1285-98, 1969.

13. ROGOT, E. \& PADGETT, S. J. Associations of coronary and stroke mortality with temperature and snowfall in selected areas of the United States, 1962-1966. Am. J. Epidemiol., 103: 565-75, 1976.

14. RUMEL, D. Indicadores de mortalidade por categoria ocupacional e nível social: Estado de São Paulo, 19801982. São Paulo, 1987. [Dissertação de Mestrado - Faculdade de Saúde Pública da USP].

15. SAVIOLA, G. A.; LUZZARI, C.; SCIPIONE, V. L.; TROLANIELLO, B.; PALOVI, A. Studio comparativo fraictus cerebrale ed andamento climatico nella provincia di Brescia. Minerva Med., 77: 2187-9, 1986.

16. TOUT, D. G. The discomfort index, mortality and the London summers of 1976 and 1978. Int. J. Biometeorol., 24: $323-8,1980$.

17. WANSTRUP, J.; KJELDSEN, K.; ASTREUP, P. Acceleration of spontaneous intimal-subintimal changes in rabbit aorta by a prolonged moderate carbon monoxide exposure. Acta Pathol. Microbiol. Scand., 75: 353-62, 1969.

Recebido para publicação em 31.8 .1992
Reapresentado em 6.11.1992

Aprovado para publicação em 9.11.1992 